\title{
Maximal Abelian Gauge and Thermal Monopoles in SU(3) gauge theory
}

\author{
Claudio Bonati \\ INFN - Sezione di Pisa, \\ Largo Pontecorvo 3, I-56127 Pisa, Italy \\ E-mail: bonatiedf.unipi.it

\section{Massimo D’Elia*} \\ Dipartimento di Fisica dell'Università di Pisa and INFN - Sezione di Pisa, \\ Largo Pontecorvo 3, I-56127 Pisa, Italy \\ E-mail: deliaedf.unipi.it
}

We discuss the extension of the Abelian projection based on the Maximal Abelian Gauge to the $S U(N)$ color gauge group. Then we investigate the properties of thermal monopoles in the high temperature phase of $S U(3)$ pure gauge theory. Some properties resemble those already found for $S U(2)$, while the presence of an enlarged abelian gauge group leads to new interesting features, related to the interaction between monopoles of different species.

QCD-TNT-III-From quarks and gluons to hadronic matter: A bridge too far?,

2-6 September, 2013

European Centre for Theoretical Studies in Nuclear Physics and Related Areas (ECT*), Villazzano, Trento (Italy)

\footnotetext{
* Speaker.
} 


\section{Introduction}

The origin of color confinement has not been clarified yet. A commonly accepted mechanism for confinement is that based on dual superconductivity of the QCD vacuum [1], 2], deriving from the condensation of magnetically charged objects. Usual ways to test this mechanism in lattice QCD simulations consists in studying the vacuum expectation value of magnetically charged operators [3, 4, 5, 6, 7, 9, 10, 8, or in looking at the properties of Abelan monopole configurations extracted from non-Abelian gauge configurations. The extraction of Abelian degrees of freedom in non-Abelian gauge theories relies on Abelian projection, which requires to choose a reference adjoint field. No natural adjoint field exists in QCD and that leads to some arbitrariness. A popular choice is to adopt the projection based on the so-called Maximal Abelian gauge (MAG).

Recently, it has been pointed out that magnetic monopoles evaporating from the low $T$ condensate may play a significant role also above deconfinement [11, 12]. Such monopoles, known as thermal monopoles, are identified, in finite $T$ lattice simulations, with the monopole currents with non-trivial wrapping in the Euclidean time direction [12, 13, 14,. Extensive studies of their properties in the $S U$ (2) Yang-Mills theory [15, 16, 17, 18, 19, 20, 21] have confirmed that the magnetic component of the Quark-Gluon Plasma is relevant especially right above the deconfinement temperature $T_{c}$, while it becomes less and less relevant in the high $T$ limit, where the theory is electrically dominated [22, 23]. Spatial correlations of thermal monopoles show the presence of screened Coulomb interactions [15], with a magnetic coupling which grows in the high $T$ regime [22]. The density of monopoles is logarithmically suppressed at high $T$, while it becomes significant as $T_{c}$ is approached from above. The statistical distribution of trajectories with multiple wrappings in the time direction [24, 16] suggests thermal monopole condensation at a temperature which coincides, within errors, with $T_{c}$, thus giving further support to the dual superconductor mechanism.

In this study we extend the investigation to $S U(3)$. The main difference with respect to $S U(2)$ is that two different species of monopoles can be identified, because the maximal Abelian subgroup of $S U(N)$ is $U(1)^{(N-1)}$. On the one hand, that makes the extension of the Maximal Abelian Gauge to $S U(3)$ less trivial; on the other hand, new properties are expected, in relation to the interactions between the two different monopole species. A full account of our results can be found in Ref. [25].

\section{Abelian projection and MAG monopoles in $S U(N)$}

Within a non-Abelian $S U(N)$ gauge theory, an Abelian 't Hooft tensor [26] can be associated with any normalized local adjoint field $\phi(x)=\sum_{a} \phi^{a}(x) T^{a}\left(\sum_{a} \phi^{a} \phi^{a}=\right.$ const.):

$$
F_{\mu v}=\operatorname{tr}\left(\phi G_{\mu v}\right)-\frac{i}{g} \operatorname{tr}\left(\phi\left[D_{\mu} \phi, D_{\nu} \phi\right]\right)
$$

In gauges where $\phi$ is constant and points along one of the $N-1$ fundamental weights (unitary gauge),

$$
\phi_{0}^{k}=\frac{1}{N} \operatorname{diag}(\underbrace{N-k, \ldots, N-k}_{k}, \underbrace{-k, \ldots,-k}_{N-k}),
$$

$F_{\mu \nu}$ reduces to a standard electromagnetic tensor (see, e.g., Refs. [27, 28]):

$$
F_{\mu \nu}^{(k)}=\operatorname{tr}\left(\partial_{\mu}\left(\phi_{0}^{k} A_{v}\right)-\partial_{v}\left(\phi_{0}^{k} A_{\mu}\right)\right) \equiv \partial_{\mu} a_{v}^{(k)}-\partial_{v} a_{\mu}^{(k)},
$$


where

$$
a_{\mu}^{(k)} \equiv \operatorname{tr}\left(\phi_{0}^{k} A_{\mu}\right)=\sum_{j=1}^{k}\left(A_{\mu}\right)_{j j}
$$

If $A_{\mu}^{D}$ is the diagonal part of the gauge field, $A_{\mu}^{a} T^{a}$, then $A_{\mu}^{D}=\sum_{k=1}^{N-1} a_{\mu}^{(k)} \alpha^{k}$ where $\alpha^{k}$ is the $k$-th simple root. Hence $F_{\mu \nu}^{(k)}$ is the electromagnetic tensor for the $U(1)$ subgroup generated by $\alpha^{k}$.

In $S U(N)$ gauge theories one can identify $N-1$ independent $U(1)$ subgroups. The standard procedure [29] is to identify a local, hermitian adjoint operator $X(x)$, i.e. $X(x) \rightarrow G(x) X(x) G^{-1}(x)$ under gauge transformations, then fixing the gauge where $X(x)$ is diagonal

$$
X(x)=X^{D}(x)=\operatorname{diag}\left(X_{1}(x), X_{2}(x), \ldots, X_{N}(x)\right) .
$$

with a given ordering for its eigenvalues [29]: $X_{j}(x) \geq X_{j+1}(x)$. That fixes the gauge apart from a residual $U(1)^{(N-1)}$ gauge group. A 't Hooft tensor $F_{\mu \nu}^{(k)}$ can then be associated with any residual $U(1)$ group, fixing $\phi(x)=\phi_{0}^{k}$ in the diagonal gauge; all $U(1)$ subgroups are mutually neutral, i.e. they are independent of each other. $X(x)$ can be taken traceless, then one can write $X^{D}(x)$

$$
X^{D}(x)=\sum_{k=1}^{N-1} c^{k}(x) \phi_{0}^{k}
$$

where $c^{k}(x)=\frac{1}{2} \operatorname{tr}\left(\alpha^{k} X^{D}(x)\right)=X_{k}(x)-X_{k+1}(x)$. A special role is played by points $x_{0}$ where $c^{k}\left(x_{0}\right)=0$ for some $k$, i.e. where $X_{k}(x)=X_{k+1}(x)$ : in $x_{0}$ the residual gauge symmetry is enlarged to the full $S U(2)$ group associated with the simple root $\alpha^{k}$. Around $x_{0}$ one can choose either a hedgehog solution [26, 30] or, in the unitary gauge, a solution where $X(x)$ is diagonal and $a_{\mu}^{(k)}$ contains the contribution from a Coulomb-like magnetic field centered around $x_{0}$. We identify $x_{0}$ as the location of a magnetic monopole for the corresponding $U(1)$ subgroup.

Let us now discuss the lattice formulation [31]. Also in this case the Abelian projection is defined in terms of a local adjoint operator $X(n)$, where the index $n$ labels the lattice sites. Having fixed the diagonal, unitary gauge, one takes the phases $\operatorname{diag}\left(\phi_{\mu}^{1}(n), \phi_{\mu}^{2}(n), \ldots \phi_{\mu}^{N}(n)\right)$ of the diagonal part of each gauge link $U_{\mu}(n)$, and then constructs the Abelian gauge phases $\theta_{\mu}^{k}(n)$, following Eq. (2.4), as

$$
\theta_{\mu}^{k}(n)=\sum_{j=1}^{k} \phi_{\mu}^{j}(n)
$$

and finally the $k$-th 't Hooft tensor (Abelian plaquette) is constructed as

$$
\exp \left[i \theta_{\mu v}^{k}(n)\right]=\exp \left[i\left(\theta_{\mu}^{k}(n)+\theta_{v}^{k}(n+\hat{\mu})-\theta_{\mu}^{k}(n+\hat{v})-\theta_{v}^{k}(n)\right)\right] .
$$

Regarding magnetic monopoles, the recipe of locating points where one of the coefficients $c^{k}$ vanishes is not well defined on a discrete lattice, hence the standard procedure is to look for points from which, in the unitary gauge, a net magnetic flux comes out (De Grand-Toussaint construction [32]). In particular, monopole currents of are defined as

$$
m_{\mu}^{k}=\frac{1}{2 \pi} \varepsilon_{\mu \nu \rho \sigma} \hat{\partial}_{v} \bar{\theta}_{\rho \sigma}^{k}
$$


where $\hat{\partial}_{v}$ is the discretized derivative and $\bar{\theta}_{\rho \sigma}^{k}$ is the compactified abelian plaquette phase

$$
\theta_{\mu \nu}^{k}=\bar{\theta}_{\mu \nu}^{k}+2 \pi n_{\mu \nu}^{k}, \quad n_{\mu \nu}^{k} \in \mathbf{N}, \quad \bar{\theta}_{\mu \nu}^{k} \in[0,2 \pi) .
$$

A standard choice is to define the Abelian projection in the so-called Maximal Abelian Gauge (MAG) [33]. For $S U(2)$, MAG is defined as the gauge which maximizes

$$
F_{\mathrm{MAG}}=\sum_{\mu, n} \operatorname{tr}\left(U_{\mu}(n) \sigma_{3} U_{\mu}^{\dagger}(n) \sigma_{3}\right) .
$$

$F_{\text {MAG }}$ is proportional, apart from a constant term, to the sum of the squared diagonal elements of the gauge links. On stationary points of $F_{\mathrm{MAG}}$, the traceless hermitean operator

$$
X^{\mathrm{MAG}}(n)=\sum_{\mu}\left[U_{\mu}(n) \sigma_{3} U_{\mu}^{\dagger}(n)+U_{\mu}^{\dagger}(n-\mu) \sigma_{3} U_{\mu}(n-\mu)\right],
$$

is diagonal and can be taken, for MAG fixed configurations, as the local operator which defines the Abelian projection, while in a generic gauge it is obtained by requiring that it transforms as a local adjoint field. Part of the popuparity of the MAG projection is due to the fact that abelian projected fields retain most of the original dynamics (Abelian Dominance). The properties of magnetic monopoles defined after MAG projection also show a nice scaling to the continuum limit.

A possible generalization for $S U(3)$ [35], usually adopted in the literature [36, 37, 38, 39], is to maximize the sum of the squared diagonal elements of the gauge links. However, by this extension no diagonal adjoint operator can be naturally identified. Morover, on extremal points, the residual symmetry is not just $U(1)^{(N-1)}$, since global permutations of group indexes leave the functional invariant. Such permutations mix the two Abelian charges, which then become ill-defined.

An alternative possibility [40, 41, 42, 25] is the following

$$
\tilde{F}_{\mathrm{MAG}}=\sum_{\mu, n} \operatorname{tr}\left(U_{\mu}(n) \tilde{\lambda} U_{\mu}^{\dagger}(n) \tilde{\lambda}\right) ; \quad \tilde{\lambda}=\operatorname{diag}\left(\tilde{\lambda}_{1}, \tilde{\lambda}_{2}, \ldots \tilde{\lambda}_{N}\right),
$$

where $\tilde{\lambda}$ is a generic element of the Cartan subalgebra. A number of properties can be shown (see Ref. [25]). A diagonal Higgs field exists, provided $\tilde{\lambda}$ has no pair of coinciding eigenvalues

$$
\tilde{X}(n)=\sum_{\mu}\left[U_{\mu}(n) \tilde{\lambda} U_{\mu}^{\dagger}(n)+U_{\mu}^{\dagger}(n-\mu) \tilde{\lambda} U_{\mu}(n-\mu)\right] .
$$

If we require $X_{j}(n) \geq X_{j+1}(n)$ around the perturbative vacuum, then the condition reads

$$
\tilde{\lambda}=\sum_{k=1}^{N-1} b^{k} \phi_{0}^{k} ; \quad b^{k}>0 \quad \forall k
$$

and the residual symmetry is strictly $U(1)^{(N-1)}$. That also guarantees that no pair of coinciding eigenvalues can appear around the perturbative vacuum, where $X(n)=\sum_{k=1}^{N-1} c^{k}(n) \phi_{0}^{k}$ with $c^{k} \sim b^{k}$, hence the appearance of monopoles requires non-perturbative fluctuations. In the following, we will adopt $b^{k}=1 \forall k$, which treats all monopole species symmetrically:

$$
\tilde{\lambda}=\sum_{k=1}^{N-1} \phi_{0}^{k}=\operatorname{diag}\left(\frac{N-1}{2}, \frac{N-1}{2}-1, \frac{N-1}{2}-2, \ldots,-\frac{N-1}{2}\right) .
$$




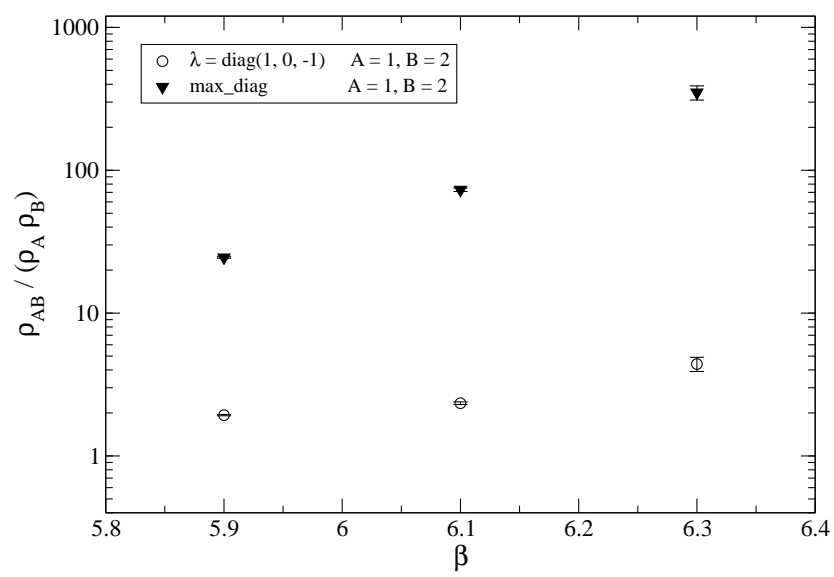

Figure 1: Probability of two coinciding monopole currents, normalized by the corresponding single current probabilities, for different values of $\beta$ and for different choices of the monopoles currents.

To summarize, for $S U(3)$, we consider the MAG corresponding to the functional defined by $\tilde{\lambda}=\operatorname{diag}(1,0,-1)$, we take the diagonal part of gauge links, $U_{\mu}^{D}(n)=\operatorname{diag}\left(e^{i \phi_{\mu}^{1}(n)}, e^{i \phi_{\mu}^{2}(n)}, e^{i \phi_{\mu}^{3}(n)}\right)$, and determine the Abelian phases $\theta_{\mu}^{1}(n), \theta_{\mu}^{2}(n)$, corresponding to the two residual $U(1)$ subgroups $\theta_{\mu}^{1}(n)=\phi_{\mu}^{1}(n)$ and $\theta_{\mu}^{2}(n)=-\phi_{\mu}^{3}(n)$ (see Eq. (2.7)). Starting from $\theta_{\mu}^{1}(n)$ and $\theta_{\mu}^{2}(n)$, we determine the two monopole currents $m_{\mu}^{1}$ and $m_{\mu}^{2}$. Monopole currents form closed loops, since $\hat{\partial}_{\mu} m_{\mu}^{k}=0$, and we are interested in particular in loops with a non-trivial wrapping around the Euclidean time direction, which are identified with thermal monopoles [12, 13, 14, 15].

It is interesting to give a quantitative estimate about the "independence" of the two monopole species. Let us call $\rho_{A}\left(\rho_{B}\right)$ the probability that a given three-dimensional cube of the lattice is pierced by monopole current $m_{\mu}^{A}\left(m_{\mu}^{B}\right)$, and by $\rho_{A B}$ the probability that the cube is pierced by both currents. If the two currents are independent of each other, one expects $\rho_{A B} / \rho_{A} \rho_{B} \sim 1$, apart from small corrections due to physical interactions between them. We have studied $\rho_{A B} / \rho_{A} \rho_{B}$ on a $16^{4}$ lattice, with the Wilson action and for a few values of the inverse gauge coupling $\beta$, adopting different choices of the monopole currents, $m_{\mu}^{A}$ and $m_{\mu}^{B}$, corresponding to our definition of MAG and to the standard definition. Results are reported in Fig. 1. In the first case the ratio is $O(1)$ for all explored values of $\beta$, as expected for independent currents. On the other hand, when one fixes the gauge by maximizing the sum of the squared diagonal elements of gauge links, the overlap between the two currents is clearly visible and $\rho_{A B} /\left(\rho_{A} \rho_{B}\right) \gg 1$ for all the explored values of $\beta$.

It is well known that functionals like that in Eq. (2.13) possess many local maxima, corresponding to different Gribov copies. In the case of our interest, every local maximum of the functional $\tilde{F}_{\mathrm{MAG}}$ in Eq. (2.13) will lead to a well defined diagonal operator $\tilde{X}(n)$, hence to a legitimate Abelian projection. We will take the Gribov copy which is found first by the maximization procedure, starting from the original configuration sampled by the Monte-Carlo algorithm: this is a practical criterion which leads to a correct scaling in the continuum limit [15]. Accurate studies have been performed for $S U(2)$ thermal monopoles, adopting simulated annealing procedures in order to get as close as possible to the global maximum of the MAG functional [43, 18, 19]: apart from a $20 \%$ difference in the monopole density, no other significant differences have been found. 

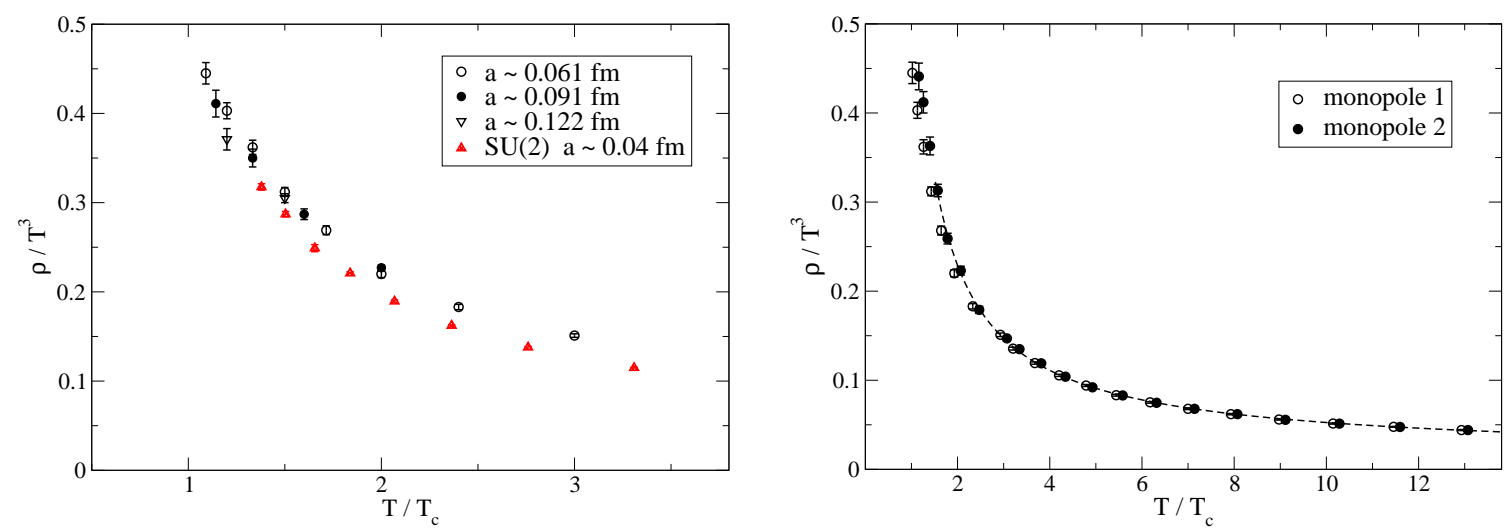

Figure 2: Left: thermal monopole density for various lattice spacings and comparison with $S U(2)$ data. Right: monopole densities over an extended temperature range together with a fit to Eq. 3.2.

In the future, one should consider other gauge conditions, like the Laplacian gauge [44], which are safe from ultraviolet fluctuations as the MAG is, but are also free of Gribov copy ambiguities.

\section{Numerical results}

We will present and discuss results obtained for the $S U(3)$ pure gauge theory discretized with the Wilson plaquette action and simulated by a standard combination of heat-bath [45] and overrelaxation [46] algorithms. The temperature $T=1 /\left(L_{t} a(\beta)\right)$ has been tuned by changing both the number of temporal sites, $L_{t}$, at fixed lattice spacing and by tuning the inverse bare coupling $\beta$ at fixed $L_{t}$. A number of different spatial sizes have been explored, ranging from $24^{3} \times L_{t}$ to $48^{3} \times L_{t}$. In order to obtain the temperature $T$ in units of $T_{c}$, we have made use of the nonperturbative determination of $a(\beta)$ and of the critical $\beta$ values reported in Ref. [47] for various values of $L_{t}$. For each parameter set, thermal monopole trajectories have been determined on a number of decorrelated configurations ranging from a few hundreds up to a few thousands.

In order to identify thermal monopoles on a given gauge configuration, we first look for monopole currents piercing a given time slice (e.g., at $t=0)$ in the temporal direction, then we follow the current and keep trace of the number of temporal wrappings that the current makes before coming back to the original detection point. Currents wrapping in the positive or negative directions are associated respectively with monopoles or antimonopoles. If the current wraps 2 or more times, then it can be associated with 2 or more thermal monopoles undergoing a cyclic permutation as they go around the thermal cycle: such trajectories are characteristic of the path integral representation of a system of identical, bosonic particles.

A first quantity that we will look at is the total density of thermal monopoles. For a given species that is defined as [12, 13, 14]

$$
\rho=\frac{\left\langle\sum_{\vec{n}}\left|N_{\text {wrap }}\left(m_{0}(\vec{n}, t)\right)\right|\right\rangle}{V_{s}}
$$

where $N_{\text {wrap }}\left(m_{0}(\vec{n}, t)\right)$ is the time winding number of the monopole current starting from $m_{0}$ at the lattice site $(\vec{n}, t)$, while $V_{s}=\left(L_{s} a\right)^{3}$ is the spatial volume. It is convenient to consider the 

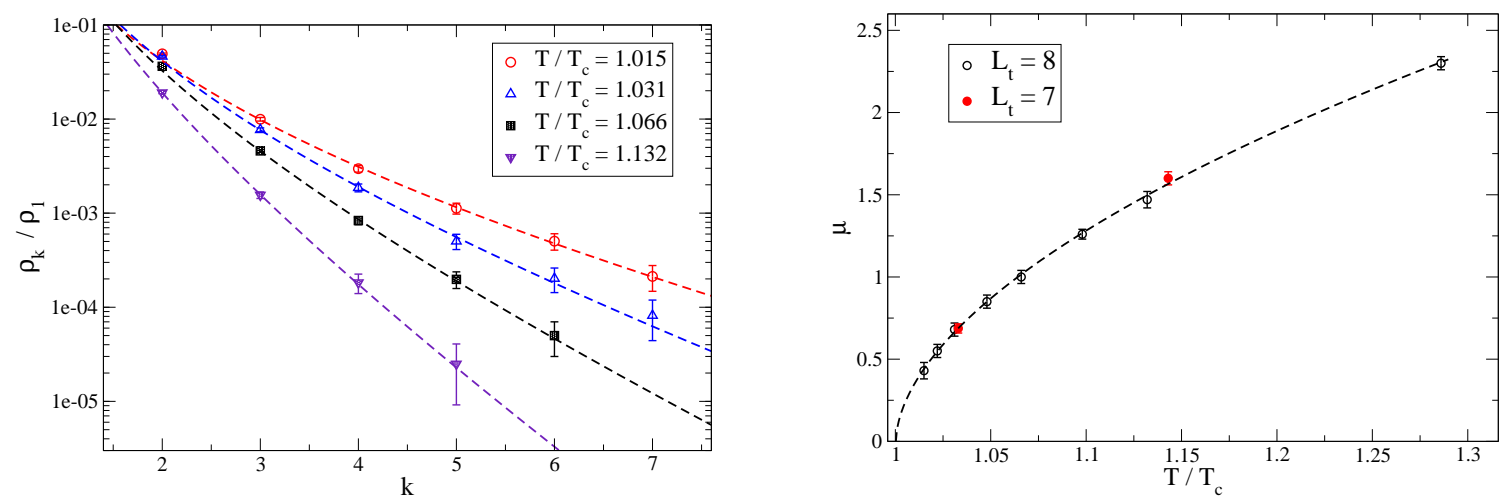

Figure 3: Left: normalized densities of trajectories with $k$ wrappings, together with fits according to Eq. 3.3. . Right: chemical potentials obtained at various temperatures and lattice spacings, together with a fit to Eq. (3.4).

dimensionless ratio $\rho / T^{3}$, which for a gas of free quantum particles and antiparticles of mass $m$ should approach a constant value as $T \gg m$.

In Fig. Q, left, we show $\rho / T^{3}$ for the first monopole species and for different values of $T$ and $a$; results obtained for the second species are compatible within errors. We notice a good scaling to the continuum limit. No significant deviations are observed with respect to the data obtained for $S U(2)$ in Ref. [15], which are reported in the figure as well. In Fig. Q, right, we report the densities of both monopoles over an extended temperature range. Contrary to the expectation for a gas of free particles, $\rho / T^{3}$ does not approach a constant behavior, even at asymptotically high $T$; this is in agreement with a scenario in which the high $T$ phase is electrically dominated, while the magnetic component is strongly interacting [11]. In particular, perturbative and dimensional reduction considerations predict that $\rho / T^{3}$ decreases like $g^{6}$ [48, 11], where $g(T)$ is the renormalized running coupling, so that we have tried to fit data according to

$$
\frac{\rho}{T^{3}}=\frac{A}{\left(\log \left(T / \Lambda_{e f f}\right)\right)^{3}},
$$

which works well for all data at $T / T_{c} \geq 2$, with $\chi^{2} /$ d.o.f. $=9.5 / 13$. The corresponding fit function is shown in the figure.

Further information can be obtained by looking at the density of trajectories with multiple wrappings. They can be related to the properties of monopoles as identical quantum particles. Indeed, the path integral representation of the partition function of $N$ identical particles is made up of path configurations which are periodic apart from possible permutations of the $N$ particles. Each configuration presents in general $M$ closed paths, with $M \leq N$ and the $j$-th path wrapping $k_{j}$ times, so that that $\sum_{j=1}^{M} k_{j}=N$ : such configuration derives from a permutation made up of $M$ cycles of sizes $k_{1}, k_{2}, \ldots k_{M}$.

Large cycles are exponentially suppressed at high $T$, where the system is close to the Boltzmann approximation and configurations deviating from the identical permutation give a negligible weight to the path integral. Their statistical weight instead increases as quantum effects become 

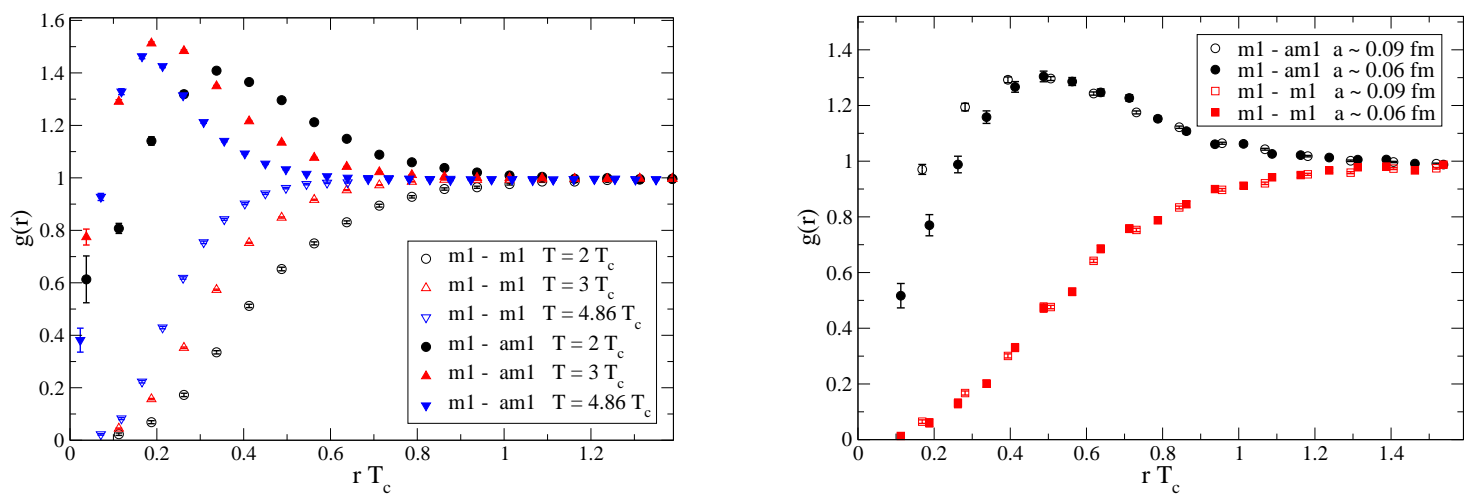

Figure 4: Left: correlation function $g(r)$ between monopoles and (anti)monopoles of the same species for various temperatures. Right: as on the left, for $T=1.33 T_{c}$ and different values of the lattice spacing.

important, and in a critical way as one approaches phenomena like Bose-Einstein condensation (BEC) [49]. For a set a free bosons of mass $m$, in particular, one finds that the density of paths wrapping $k$ times ( $k$-cycles) is [50]:

$$
\rho_{k} \equiv \frac{\left\langle n_{k}\right\rangle}{V_{s}}=\frac{e^{-\hat{\mu} k}}{\lambda^{3} k^{5 / 2}}
$$

where $n_{k}$ is the number of $k$-cycles in one configuration, $\lambda=\sqrt{2 \pi /(m T)}$ is the De Broglie thermal wavelength, and $\hat{\mu} \equiv-\mu / T$ where $\mu$ is the chemical potential. As $\hat{\mu} \rightarrow 0$, higher $k$-cycles become more and more frequent and the system approaches criticality.

In Fig. 3, left, we report the density of trajectories wrapping $k$ times, $\rho_{k}$, normalized by $\rho_{1}$, as a function of $k$ for a few values of $T$, as obtained by simulations on a $48^{3} \times 8$ lattice. For each $k$ the relative weight of $\rho_{k}$ rapidly increases as $T$ approaches $T_{c}$. Moreover, as it happens for $S U(2)$ [16], data can be nicely fitted according to the ansatz in Eq. (3.3), giving us access to an effective value of $\hat{\mu}(T)$. The effective chemical potentials are reported in Fig. 3 , right, where also results from a $48^{3} \times 7$ lattice are shown, in order to check for a correct scaling to the continuum limit.

Following Ref. [16], we have fitted $\hat{\mu}(T)$ according to a critical behavior:

$$
\hat{\mu}=A\left(T-T_{\mathrm{BEC}}\right)^{v^{\prime}}
$$

obtaining $A=4.64(20), v^{\prime}=0.56(3)$ and $T_{\mathrm{BEC}}=1.0003(36) T_{c}$, with $\chi^{2} /$ d.o.f. $=1.5 / 7$, including all available data. Therefore, also in the $S U(3)$ pure gauge theory the distribution of wrapping trajectories supports a BEC-like phenomenon for magnetic charges happening right at $T_{c}$, in agreement with a dual superconductor scenario. Notice that for $S U(3)$, since the transition is first order, the coincidence of $T_{c}$ with $T_{\mathrm{BEC}}$ extracted according to Eq. (3.4) is not expected apriori, since the transition is first order; in particular, one would expect $T_{\mathrm{BEC}} \leq T_{c}$. However the transition for $S U(3)$ is quite weak and that could explain the observed coincidence. It will be interesting to repeat the same analysis for $S U(N)$ gauge theories with $N>3$, where the transition gets stronger.

Finally, let us look at the spatial distribution of thermal monopoles at a given Euclidean time slice, which gives information about the mutual interactions among such objects. In particular, we 


\begin{tabular}{|c|c|c|c|}
\hline$T / T_{c}$ & $\alpha_{M}$ & $\lambda_{P} T_{c}$ & $\chi^{2} /$ d.o.f. \\
\hline 1.333 & $3.1(4)$ & $0.285(11)$ & $52 / 36$ \\
\hline 2 & $4.3(5)$ & $0.209(6)$ & $48 / 39$ \\
\hline 3 & $5.9(5)$ & $0.151(3)$ & $48 / 41$ \\
\hline 4.86 & $6.4(6)$ & $0.114(3)$ & $47 / 41$ \\
\hline
\end{tabular}

Table 1: Parameters of the interaction potential in Eq. (3.7) for various temperatures, obtained by a common fit to monopole-monopole and monopole-antimonopole spatial correlations of the same species.

will study the density-density correlation function $g_{A B}(r) \equiv\left\langle\rho^{A}(0) \rho^{B}(r)\right\rangle /\left(\rho^{A} \rho^{B}\right)$ between any couple $A$ and $B$ of thermal monopole species. That is defined as the ratio between the probability of having a monopole of species $B$ at distance $r$ from a given reference monopole of species $A$, and the same probability in case the monopoles are uncorrelated and randomly distributed, i.e.

$$
g_{A B}(r)=\frac{1}{\rho^{B}} \frac{d N^{B}(r)}{4 \pi r^{2} d r}
$$

where $d N^{B}(r)$ is the number of monopoles in a spherical shell of thickness $d r$ and radius $r$ around the reference monopole; in order to minimize lattice artifacts we have used, in place of $4 \pi r^{2} d r$, the effective number of lattice sites contained in the shell. A value $g_{A B}(r)<1\left(g_{A B}(r)>1\right)$ indicates a repulsive (attractive) interaction.

In Fig. 4 we show the correlation functions among monopoles and antimonopoles of the same species and for three different temperatures (left), while an additional temperature value is shown on the right, where data for two different lattice spacings are shown in order to check for the correct scaling to the continuum limit. Results are qualitatively similar to those obtained for $S U(2)$; a qualitative agreement is also found with the results for monopole-monopole interactions reported in Ref. [51], which are based on the standard extension of MAG to $S U(3)$.

As for $S U(2)$, we have extracted the potential $V(r)$ by looking at the large distance region, where

$$
g_{A B}(r) \simeq \exp \left(-V_{A B}(r) / T\right) .
$$

and then fitted it according to a screened Coulomb potential,

$$
V_{A B}(r)=\frac{\alpha_{M} e^{-\lambda_{P} r}}{r}
$$

We have tried a common fit to monopole-monopole and monopole-antimonopole correlations, in order to check whether they can be described by the same magnetic coupling: $\chi^{2}$ values are marginally acceptable and are reported, together with fit results, in Table 1. As for $S U(2)$, the magnetic coupling $\alpha_{M}$ shows a slowly increasing behavior with $T$, in agreement with arguments based on the electric-magnetic duality [11]; a rough agreement is also obtained with the results of Ref. [51]. The screening length instead decreases with $T$. The so-called plasma parameter $\Gamma \equiv \alpha_{M}\left(4 \pi \rho / 3 T^{3}\right)^{1 / 3}$ stays always well above 1 , as expected for a strongly interacting plasma.

A new aspect of $S U(3)$, with respect to $S U(2)$, is that we can study correlations among different monopole species, $g_{m^{1} m^{2}}(r)$ and $g_{m^{1}} a_{m^{2}}(r)$. Those are shown in Fig. 5, left, for two different 

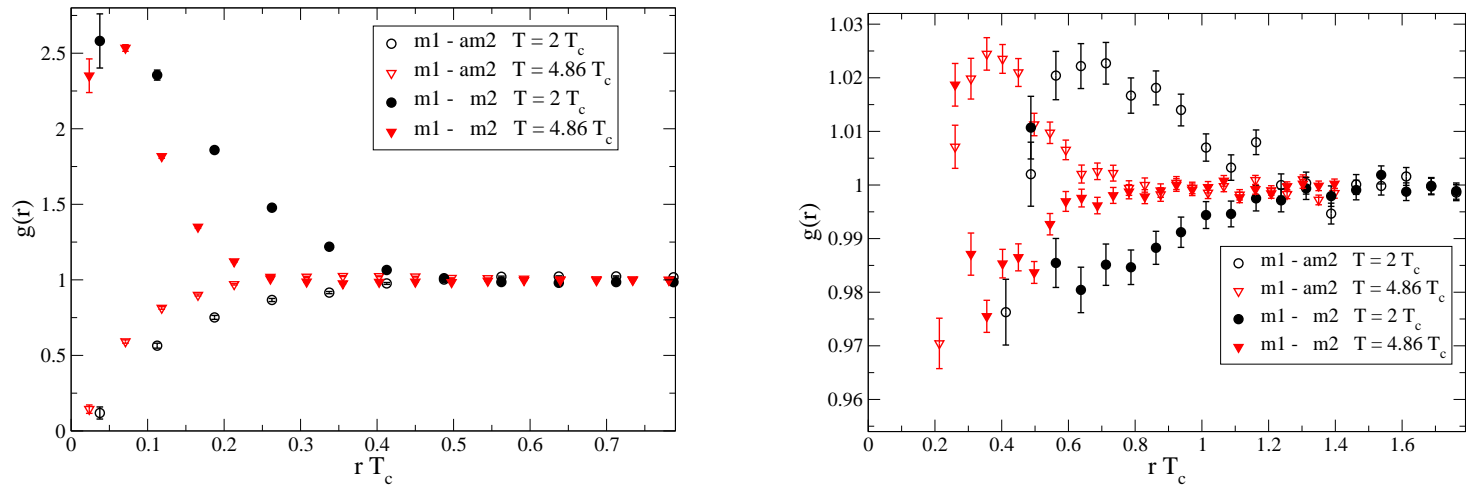

Figure 5: Left: correlation function $g(r)$ between monopoles and (anti)monopoles of different species for various temperatures. Right: as on the left, with a zoom on the intermediate distance region.

values of $T$. Correlations are similar to those among monopoles of the same species, but the sign is opposite, with attraction (repulsion) between monopoles and (anti)monopoles of different species. This is not unexpected, since the magnetic charge operators of each species are proportional to the corresponding roots, i.e. $\lambda_{3}=\operatorname{diag}(1,-1,0) / 2$ and $\lambda_{3}^{\prime}=\operatorname{diag}(0,1,-1) / 2$ for $S U(3)$, hence the mutual interaction must be proportional to $\operatorname{Tr}\left(\lambda_{3}^{\prime} \lambda_{3}\right)=-1 / 4$ (see, e.g. , Ref. [52]), while in the case of same species monopoles the interaction is proportional to $\operatorname{Tr}\left(\lambda_{3}^{2}\right)=\operatorname{Tr}\left(\lambda_{3}^{\prime 2}\right)=1 / 2$.

New, unexpected features however appear after a more careful observation, as shown in the right hand side of Fig. 5, where the intermediate distance region has been magnified. After the first positive peak in $g_{m^{1} m^{2}}(r)$, a small negative well develops for $g-1$, corresponding to a repulsive interaction at intermediate distances; the opposite behavior is visible also in the monopoleantimonopole correlation, $g_{m^{1} a m^{2}}(r)$. An oscillating behavior of $g(r)$ is typical of systems with partial ordering, like liquids. A possible explanation could be that monopoles of different species are bound into larger objects (think of calorons and of their monopole constituents), and that such larger objects weakly repel each other.

It is tempting to relate thermal monopoles to caloron constituents carrying fractional topological charges [53], and to make a connection between thermal monopole condensation and the drastic change in $\theta$ dependence observed at the deconfining transition [54, 55, 56, 57, 58, 59]. All that claims for an extension of this study to $S U(N)$ gauge theories, with $N>3$, where the interaction pattern should be even more interesting.

\section{Acknowledgments}

We thank A. Di Giacomo, E. Shuryak and A. Zhitnitsky for many useful discussions. We acknowledge the use of the computer facilities of the INFN CSNIV cluster in Pisa.

\section{References}

[1] G. 't Hooft, in "High Energy Physics", EPS International Conference, Palermo 1975, ed. A. Zichichi.

[2] S. Mandelstam, Phys. Rept. 23 (1976) 245. 
[3] L. Del Debbio, A. Di Giacomo, G. Paffuti and P. Pieri, Phys. Lett. B 355 (1995) 255 [hep-lat/9505014].

[4] A. Di Giacomo, B. Lucini, L. Montesi, G. Paffuti, Phys. Rev. D 61 (2000) 034503 [arXiv:hep-lat/9906024]; Phys. Rev. D 61 (2000) 034504 [arXiv:hep-lat/9906025];

[5] J. M. Carmona, M. D’Elia, A. Di Giacomo, B. Lucini, G. Paffuti, Phys. Rev. D 64 (2001) 114507 [arXiv:hep-lat/0103005];

[6] J. M. Carmona, M. D’Elia, L. Del Debbio, A. Di Giacomo, B. Lucini, G. Paffuti, Phys. Rev. D 66 (2002) 011503 [arXiv:hep-lat/0205025];

[7] M. D’Elia, A. Di Giacomo, B. Lucini, G. Paffuti, C. Pica, Phys. Rev. D 71 (2005) 114502 [arXiv:hep-lat/0503035].

[8] C. Bonati, G. Cossu, M. D’Elia and A. Di Giacomo, Phys. Rev. D 85 (2012) 065001 [arXiv:1111.1541 [hep-lat]].

[9] M. N. Chernodub, M. I. Polikarpov and A. I. Veselov, Phys. Lett. B 399 (1997) 267.

[10] P. Cea and L. Cosmai, JHEP 0111 (2001) 064; P. Cea, L. Cosmai and M. D’Elia, JHEP 0402 (2004) 018 .

[11] J. Liao, E. Shuryak, Phys. Rev. C 75 (2007) 054907 [hep-ph/0611131].

[12] M. N. Chernodub and V. I. Zakharov, Phys. Rev. Lett. 98 (2007) 082002 [arXiv:hep-ph/0611228]; M. N. Chernodub and V. I. Zakharov, arXiv:hep-ph/0702245.

[13] V.G. Bornyakov, V.K. Mitrjushkin and M. Muller-Preussker Phys. Lett. B 284 (1992) 99.

[14] S. Ejiri, Phys. Lett. B 376 (1996) 163 [arXiv:hep-lat/9510027].

[15] A. D’Alessandro and M. D’Elia, Nucl. Phys. B 799 (2008) 241 [arXiv:0711.1266 [hep-lat]].

[16] A. D’Alessandro, M. D’Elia and E. V. Shuryak, Phys. Rev. D 81 (2010) 094501 [arXiv:1002.4161 [hep-lat]].

[17] M. N. Chernodub, A. D’Alessandro, M. D’Elia and V. I. Zakharov, arXiv:0909.5441 [hep-ph].

[18] V. G. Bornyakov and V. V. Braguta, Phys. Rev. D 84 (2011) 074502 [arXiv:1104.1063 [hep-lat]].

[19] V. G. Bornyakov and A. G. Kononenko, Phys. Rev. D 86 (2012) 074508 [arXiv:1111.0169 [hep-lat]].

[20] V. G. Bornyakov and V. V. Braguta, Phys. Rev. D 85 (2012) 014502 [arXiv:1110.6308 [hep-lat]].

[21] V. V. Braguta and A. Y. Kotov, Phys. Rev. D 86 (2012) 014511 [arXiv:1208.5344 [hep-lat]].

[22] J. Liao and E. Shuryak, Phys. Rev. Lett. 101 (2008) 162302 [arXiv:0804.0255 [hep-ph]].

[23] C. Ratti and E. Shuryak, Phys. Rev. D 80 (2009) 034004 [arXiv:0811.4174 [hep-ph]].

[24] M. Cristoforetti and E. Shuryak, Phys. Rev. D 80 (2009) 054013 [arXiv:0906.2019 [hep-ph]].

[25] C. Bonati and M. D’Elia, Nucl. Phys. B 877 (2013) 233 [arXiv:1308.0302 [hep-lat]].

[26] G. 't Hooft, Nucl. Phys. B 79 (1974) 276.

[27] L. Del Debbio, A. Di Giacomo, B. Lucini and G. Paffuti, hep-lat/0203023.

[28] A. Di Giacomo, L. Lepori and F. Pucci, JHEP 0810 (2008) 096 [arXiv:0810.4226 [hep-lat]].

[29] G. 't Hooft, Nucl. Phys. B 190 (1981) 455.

[30] A. M. Polyakov, JETP Lett. 20 (1974) 194. 
[31] A. S. Kronfeld, G. Schierholz and U. J. Wiese, Nucl. Phys. B 293 (1987) 461.

[32] A. De Grand, D. Toussaint, Phys. Rev. D 22 (1980) 2478.

[33] A. S. Kronfeld, M. L. Laursen, G. Schierholz, U.-J. Wiese Phys. Lett. B 198 (1987) 516.

[34] C. Bonati, A. Di Giacomo and M. D’Elia, Phys. Rev. D 82 (2010) 094509 [arXiv:1009.2425 [hep-lat]].

[35] F. Brandstaeter, G. Schierholz, U.-J. Wiese Phys. Lett. B 272 (1991) 319.

[36] W. W. Tucker and J. D. Stack, Nucl. Phys. Proc. Suppl. 106 (2002) 643 [hep-lat/0110165].

[37] V. Bornyakov et al., Nucl. Phys. Proc. Suppl. 106 (2002) 634 [hep-lat/0111042].

[38] V. G. Bornyakov et al. [DIK Collaboration], Phys. Rev. D 70 (2004) 054506 [hep-lat/0401026].

[39] V. G. Bornyakov et al. [DIK Collaboration], Phys. Rev. D 70 (2004) 074511 [hep-lat/0310011].

[40] J. D. Stack, W. W. Tucker, R. J. Wensley Nucl. Phys. B 639 (2002) 203 [hep-lat/0110196].

[41] J. D. Stack, W. W. Tucker and R. J. Wensley, hep-lat/0205006.

[42] W. W. Tucker and J. D. Stack, Nucl. Phys. Proc. Suppl. 119 (2003) 721 [hep-lat/0209134].

[43] G. S. Bali, V. Bornyakov, M. Mueller-Preussker, K. Schilling, Phys. Rev. D 54 (1996) 2863 [hep-lat/9603012]; V. G. Bornyakov, D. A. Komarov, M. I. Polikarpov, Phys. Lett. B 497 (2001) 151.

[44] C. Alexandrou, M. D’Elia and P. de Forcrand, Nucl. Phys. Proc. Suppl. 83 (2000) 437 [hep-lat/9907028]; P. de Forcrand and M. Pepe, Nucl. Phys. B 598 (2001) 557 [hep-lat/0008016].

[45] N. Cabibbo and E. Marinari, Phys. Lett. B 119 (1982) 387.

[46] M. Creutz, Phys. Rev. D 36 (1987) 515.

[47] G. Boyd et al., Nucl. Phys. B 469 (1996) 419.

[48] P. Giovannangeli and C. P. Korthals Altes, Nucl. Phys. B 608 (2001) 203 [arXiv:hep-ph/0102022].

[49] R.P. Feynman, Phys. Rev. 90 (1953) 1116; Phys. Rev. 91 (1953) 1291.

[50] V. Elser, PhD. Thesis, U.C. Berkeley, (1984)

[51] V. G. Bornyakov, A. G. Kononenko and V. K. Mitrjushkin, PoS ConfinementX (2012) 048; arXiv:1312.4085 [hep-lat].

[52] M. Unsal and L. G. Yaffe, Phys. Rev. D 78 (2008) 065035 [arXiv:0803.0344 [hep-th]].

[53] T. C. Kraan and P. van Baal, Phys. Lett. B 435 (1998) 389 [hep-th/9806034].

[54] O. Bergman and G. Lifschytz, JHEP 0704 (2007) 043 [hep-th/0612289].

[55] A. Parnachev and A. R. Zhitnitsky, Phys. Rev. D 78 (2008) 125002 [arXiv:0806.1736 [hep-ph]].

[56] A. R. Zhitnitsky, Nucl. Phys. A 813 (2008) 279 [arXiv:0808.1447 [hep-ph]]; hep-ph/0601057.

[57] C. Bonati, M. D’Elia, H. Panagopoulos and E. Vicari, Phys. Rev. Lett. 110 (2013) 252003 [arXiv:1301.7640 [hep-lat]].

[58] M. D’Elia and F. Negro, Phys. Rev. D 88 (2013) 034503 [arXiv:1306.2919 [hep-lat]].

[59] A. R. Zhitnitsky, Nucl. Phys. A 921 (2014) 1 [arXiv:1308.0020 [hep-ph]].

[60] J. E. Mandula, M. Ogilvie Phys.Lett. B 248 (1990) 156. 